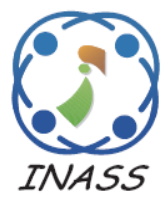

\title{
Design and Comprehensive Investigation of High Capacity Communication System Based on All Optical Orthogonal Frequency Division Multiplexing Processing
}

\author{
Ali Hayder Abdul Kareem ${ }^{1 *}$ \\ ${ }^{1}$ Ministry of Education, Director of Education in Karbala, Department of School Buildings, Iraq \\ * Corresponding author's Email: Ali_hayder@karbalaedu.org
}

\begin{abstract}
This paper introduces the concept of designing all optical orthogonal frequency division multiplexing (AO-OFDM) techniques to increase data rates to obtain a greater range of transmission. Arrayed waveguide grating (AWG) was proposed in this work as inverse fast fourier transform / fast fourier transform (IFFT/FFT) circuits in all optical orthogonal frequency division multiplexing system for its structure is simplified and capacity in large subcarriers. AWG combining with dense wave division multiplexing (DWDM) technique with $20 \mathrm{GHz}$ channel spacing. The proposed system is simpler than others, which can obtain AO-OFDM and have efficiency in the spectrum. The output investigated for the proposed system is evaluated by applying different input power and transmission lengths to assess the quality of the signal via the bit error rate (BER) parameter. The simulation results show the constellation diagram and BER of the received signal with 8 channels, using quadrature amplitude modulation (QAM) and quadrature phase shift keying (QPSK) at $(240 \mathrm{~Gb} / \mathrm{s})$ per channel.
\end{abstract}

Keywords: AO-OFDM, AWG, IFFT/ FFT, BER.

\section{Introduction}

Since a great number of devices and technological advances, a huge jump in network traffic is observed [1]. Several different methods have been suggested to increase optical network spectral efficiency and the capacity of the channel to respond to the requirements of recently advanced applications requiring large bandwidth and high data rates [2]. Orthogonal frequency division multiplexing (OFDM) is a popular technique for fast speed wireless service, like third generation, wireless cellular networks of the fourth generation, and WiMAX [3]. This is because the OFDM techniques can minimize the impact of dispersive channels by moving them to multiple flat channels and have high spectral efficiency. OFDM techniques for the optical transmission systems have recently been proposed [4], as its more efficient in channeling dispersion compared to traditional strategies as time division multiplexing [5]. These are also more effective spectrum than wavelength division multiplexing (WDM) techniques since they employ subcarrier orthogonal property [6]. OFDM techniques, furthermore, have a high peak to average power ratios and are more sensitive to both phase noise and frequency offset from the carrier [7]. OFDM systems typically using both the Fast Fourier Transform (FFT) and Inverse FFT modules to process electronically. These two modules need high speed digital signal processing, a digital-to-analog transducer, and an analog-to-digital transducer. These above procedures limit the modulation speed of the OFDM symbol. In all-optical OFDM systems, optical FFT (OFFT) is used instead of FFT electric and therefore can reach a velocity higher [8]. Also, OFFT is more effective in terms of power consumption than electrical FFT thanks to using passive parts, except time gating and tuning circuitry [9]. Usually, both FFT and IFFT are performed in conventional optical OFDM systems in the electronic domain and thus restrict the bit rate for transmission. Electronic real time IFFT and FFT 
signal processing for optical OFDM signals up to $100 \mathrm{~Gb} / \mathrm{s}$ were demonstrated [10]. This constraint looks too far-kicked to meet acceptable values for producing or receiving of multi terabit per second for the signals of OFDM. The OFDM subcarriers are created optically in the AO-OFDM system, and the IFFT / FFT processing is implemented optically using optical components [11]. As compared with conventional OFDM subcarriers, each sub-carrier is modulated with an external modulator and it carries a high data rate of information [12]. Hence, AOOFDM systems can achieve a large transmission capacity and a higher bit rate [13]. The (AO-OFDM) system uses an arrayed waveguide grating (AWG) based passive tool to perform optical fast fourier transformation to create sub-channels directly in the optical domain [14].

In this research paper, a new system has been designed and developed to improve the performance of All optical OFDM systems with different modulation formats, using $20 \mathrm{GHz}$ channel spacing AWG technology. By using the VPI transmission maker 9.5 program, multiple simulations will be performed at various transmission distances to examine the effect of the new system on OFDM signals, in a supposedly ideal transmitter-receiver network. Different simulations will be carried out adjusting input power and the form of modulation used. The advantage of the proposed AO-OFDM scheme is very simple and a proper AWG design form the pulse of the baseband accordingly, that increase total data rate and transmission distance. Furthermore, it improving BER performance for the total system that increases the quality of the received signal. The paper is presented as follows: a literature survey related to this work is reviewed in section 2 . Section 3 represented the problem formulation related to the AO-OFDM system. The principle of AO-OFDM based on AWG is presented in section 4, Section 5 detailed explanation about the proposed system. The simulation results and discussion detailed in section 6. Finally, the conclusion and suggestions for future work can be found in Section 7.

\section{Literature survey}

The researchers established other methods in All optical topics of generation and transmission. A brief overview of some significant contributions literature is provided in this section.

In [7] by using a robust detection system and an AWG, proposed a coherent AO-OFDM-based passive optical network (PON) for terabit multiples per second. This proposed solution allows the downstream data rate per device to be dramatically increased and the typical single-mode fiber scope to be expanded for future long-range applications. Five-user transmission with $25 \mathrm{~Gb} / \mathrm{s}$ quadrature phase shift keying modulation per user was demonstrated over a distance of more than $40 \mathrm{~km}$.

In [8], Based on the synthesis of several truncated sink-shaped subcarriers for each sub-band, this work proposes a banded all-optical orthogonal multiplexing frequency division (B-AO-OFDM) transmission system. In this way is to promote subband reception, while the drawback is an increase in total spectral width that causes loss in spectral efficiency. Simulation results for $800 \mathrm{~km}$ long-haul transmission distance have been shown that the BAO-OFDM scheme can increase the Q-factor of the network in a bandwidth-limited receiver network and achieve a total bit rate of to $1.8 \mathrm{~Tb} / \mathrm{s}$.

The authors presented in [9] a functional scheme is implemented to perform a fast fourier transformer in the optical domain. At speeds well beyond the limits of modern electronic processing and negligible the consumption of energy, optical FFT signal processing is carried out. This scheme allows FFT processing by $\mathrm{Tb} / \mathrm{s}$ due to is not subject to electronic speed limits. The downside of this design is that it reduces energy consumption by relying on passive optical filters which process at the cost of the transmission distance.

In [10] Based on time-lenses and coherent optical sampling for high-speed complex orthogonal frequency-division multiplexing signal detection, proposed a novel AO-OFDM scheme. This research conducted a 16/10 Gbaud quadrature phase-shift keying (QPSK) all-optical OFDM detection proofof-concept experiment with all bit error levels far below the 7\% hard-overhead error correction limit. This design did not research the effect on the transmitter signal of the input power to achieve the obtained data transfer rate of $1.5 \mathrm{~Tb}$.

In [11], this paper discusses the concepts of all optical OFDM transmission which could load data with reduced device complexity and good versatility Up to $100 \mathrm{~Gb} / \mathrm{s}$ or more. The proposed system is used to deploy a PON network with various modulation schemes such as QAM, PAM, DPSK, and are compared about their performance characteristics to choose the best one among them. This study considers the use of AWG with QAM modulation to be better for all optical broadband networks without taking into account that there is an advanced modulation technique (such as QPSK) that allows more bandwidth than in this research.

The author in [13] has been proposed all optical OFDM system with wavelet transform. An all- 
optical FFT algorithm is proposed with a cascade of delay interferometers that performs the simultaneous serial to parallel conversion and FFT counting. Subcarriers in the proposed framework are encoded using QAM signals. According to the researcher's study, QAM is superior in terms of power consumption over other subcarrier modulations. The researcher considered the inclusion of the signal using QAM modulation is the best without compared to another modulation format and using high input power that effects of the receiver signal.

In [15] implement time-domain packing techniques in orthogonal frequency division multiplexing (OFDM) systems all-optically pose the design for novel passive arrayed waveguide grating (AWG) applications. The spectral efficiency of the proposed system can be increased in long-haul fiber connections. The method proposed helps to make an integrated transceiver for high spectral performance transmissions. The result of the simulation shows that the bandwidth over $200 \mathrm{GHz}$ can achieve a capacity of $1.4 \mathrm{~Tb} / \mathrm{s}$ with a suitable design system.

The main drawbacks of the aforementioned literature in this section are not obtaining a high data transfer rate for long distance simply and inexpensively using a low bandwidth to benefit from it in increasing the number of channels. Therefore, in this study, the above obstacles were overcome by merging AWG and DWDM with advanced modulation format depending on the AO-OFDM system.

\section{Problem formulation}

New technologies such as virtual reality, highdefinition three-dimensional television, and cloud storage, demand high data levels. However, these optical high-capacity channels are fed from the lower-bit rate signals. The main problem is whether the lower-bitrate information can send up to 1000 $\mathrm{km}$ or longer to get a high data rate of more than $1 \mathrm{~Tb} / \mathrm{s}$. This proposed work aims to get an optical Fast Fourier transformer (OFFT) based on AOOFDM technology. Most communication systems have embraced this technique because of robustness against inter-symbol interference, high spectral performance, and its successful implementation. In this paper, we designed a transmitter and receiver module with two multiplexing techniques AWG and DWDM, and with a separate QAM and QPSK modulation source modulates each subcarrier on an independent optical path to boost the BER output of the received signal. In addition to that, the AWG system can be combined with transmitter IQ modulators and 90 hybrids with receiver photodetectors for achieving $\mathrm{Tb} / \mathrm{s}$ capacities with substantial signal efficiency improvements.

\section{Principle of AO-OFDM based on AWG geometry conversion}

An Arrayed Waveguide Grating (AWG) MUX / DeMUX is a planar system which has imaging and dispersion properties. It consists of I / $\mathrm{O}$ waveguides, the number of which usually equal to the number of transmitted channels [16]. A typical (AWG) consists of two free-space planar star couples, and a set of arrayed planar waveguides with lengths gradually increase, connected as illustrated in Fig. 1. In practice however, AWG design is generally complicated by adding other functional elements to compensate for polarization and temperature dependencies, and to maximize the flatness of the channel passband or minimize insertion losses and inter channel crosstalk [17].

AWG is used as the IFFT / FFT Orthogonal Frequency Division Multiplexing transmission circuits. The number of channels in a free spectral range $N_{c h}[18]$ as follow:

$$
N_{c h}=\frac{\lambda_{o} R}{n_{S} d D}, \tau=\frac{N_{c} \Delta L}{c}
$$

where $\lambda_{o}$ is the center wavelength, $R$ slab region radius, $n_{s}$ is an effective index in slab region, $\tau$ is the time difference between adjacent channels, and $N_{c}$ is the group index of effective index $n_{c}$. Next, IFFT can be accomplished by AWG circuits. The Optical IFFT circuits are paired with optical phase shifters and time delays. It can conclude that the expression of $i$ th subcarrier after IFFT in one OFDM symbol time:

$$
A_{i}(t)=\sum_{n=0}^{N-1} \exp \left(-j \theta_{n}\right) \cdot a_{i}(t-n \tau)
$$

where $A_{i}(t)$ is $i$ th port optical carrier in the input slab region and $\theta_{n}$ is the phase shift. In AWG, the increase $\Delta L$ of the arrayed waveguide, cause time

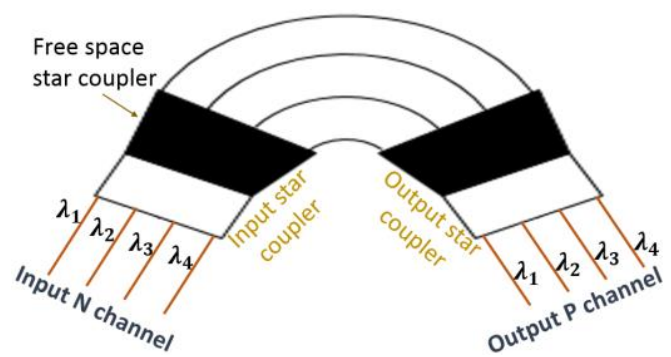

Figure. 1 Schematic view of the simplest AWG design 
delay, and the region of the input/output slab causes phase shift. For AWG, the response expresses between $i$ input and $p$ output as a filter is:

$$
\begin{aligned}
& h_{i p}(t) \\
= & \sum_{m=0}^{N-1} \exp \left(-j 2 \pi m \frac{n_{s} d}{\lambda_{o}}\left(\sin \theta_{i}+\sin \theta_{o}\right)\right) \delta(t-m \tau)
\end{aligned}
$$

where $\theta_{i}$ and $\theta_{o}$ is input and output slab region of the phase delay, and $\delta(t)$ is the Dirac delta [15]. The input/output port of the phase factor can express:

$$
\sin \theta_{i}=i \frac{D}{R} \quad \sin \theta_{o}=p \frac{D_{1}}{R}
$$

Therefore, Eq. (3) can be express from Eqs. (4) and (1)

$$
h_{i p}(t)=\sum_{m=0}^{N-1} \exp \left(-\frac{j 2 \pi m}{N_{c h}}(i+p)\right) \cdot \delta(t-m t)
$$

Then converting Eq. (5) by Fourier transformer, in spectral domain we can get the AWG transfer function:

$$
H_{i p}(f)=\sum_{m=0}^{N-1} \exp \left(-\frac{j 2 \pi m}{N_{c h}}(i+p)\right) \cdot \delta\left(-2 \pi f_{m} \Delta \tau\right)
$$

Then we can use the AWG as IFFT / FFT circuits. As signals pass through the AWG as IFFT to achieve modulation of OFDM, the express is:

$$
A_{p}(t)=\sum_{m=0}^{N-1} \exp \left(-j \frac{2 \pi m}{N_{c h}}(i+p)\right) \cdot a_{i}(t-m \tau)
$$

The output slab area contains only one port, since the signal after IFFT is the $\mathrm{N}$ subcarrier overlay. Therefore, it can select the output port where the phase shifts in the output area are equal. So the impact of $\theta_{o}$ in optical IFFT can be neglect.

$$
A_{p}(t)=\sum_{m=0}^{N-1} \exp \left(-\frac{j 2 \pi i m}{N_{c h}}\right) \cdot a_{i}(t-m \tau)
$$

Also, the optical FFT is similar in form, so, the OFDM signal can be expressed after $p$ th OFFT module.

$$
\begin{gathered}
S_{i}(t)=\sum_{m=0}^{N-1} \sum_{n=0}^{N-1} \exp \left(-j 2 \pi i \frac{(m+n)}{N_{c h}}\right) \cdot a_{i}(t \\
-(m+n) \cdot \tau)
\end{gathered}
$$

The authentic signal forms OFDM after the OFFT module.

\section{Description of the proposed system}

The concept of all optical OFDM systems is illustrated in Fig. 2. The efficiency of the scheme depends on the modulation of the subcarrier, optical gate stability and the accuracy of the passive system performing the optical FFT. The simulation parameter of the proposed system is given in Table 1. The system is consists of two parts:

\subsection{Transmission demonstration of all optical OFDM}

The transmitter subsystem consists of an optical comb, AWG, optical modulator, and an optical multiplexer. Comb generation is necessary for AOOFDM systems, due to different subcarriers must be produced from the same laser source to maintain the orthogonal between the OFDM subcarriers. The signal passed through the AWG performing the IFFT is modulated by quadrature modulator to OFDM signals. An IQ modulator consists of two Mach Zehnder modulator (MZM) with two orthogonal components produces an optical (QAM, QPSK) modulation signal. In the upper arm, the intricate envelope's in-phase component modulates the optical carrier, while in the lower arm; the quadrature-phase component modulates an optical carrier shifted by $90^{\circ}$. Following the modulation of the optical OFDM subcarriers, it will be aggregated to form the optical OFDM signal by an optical multiplexer. To keep the OFDM signals orthogonal, OFDM symbol duration is set to $T_{s}=1 / \Delta f_{s}$, where $\Delta f_{s}$ is the frequency spacing of the comb. After that, all signals are multiplexed by DWDM with $20 \mathrm{GHz}$ channel spacing.

\subsection{Receiver demonstration of all optical OFDM}

The receiver side consists of optical FFT, DWDM demultiplexer, and optical demodulator. All 


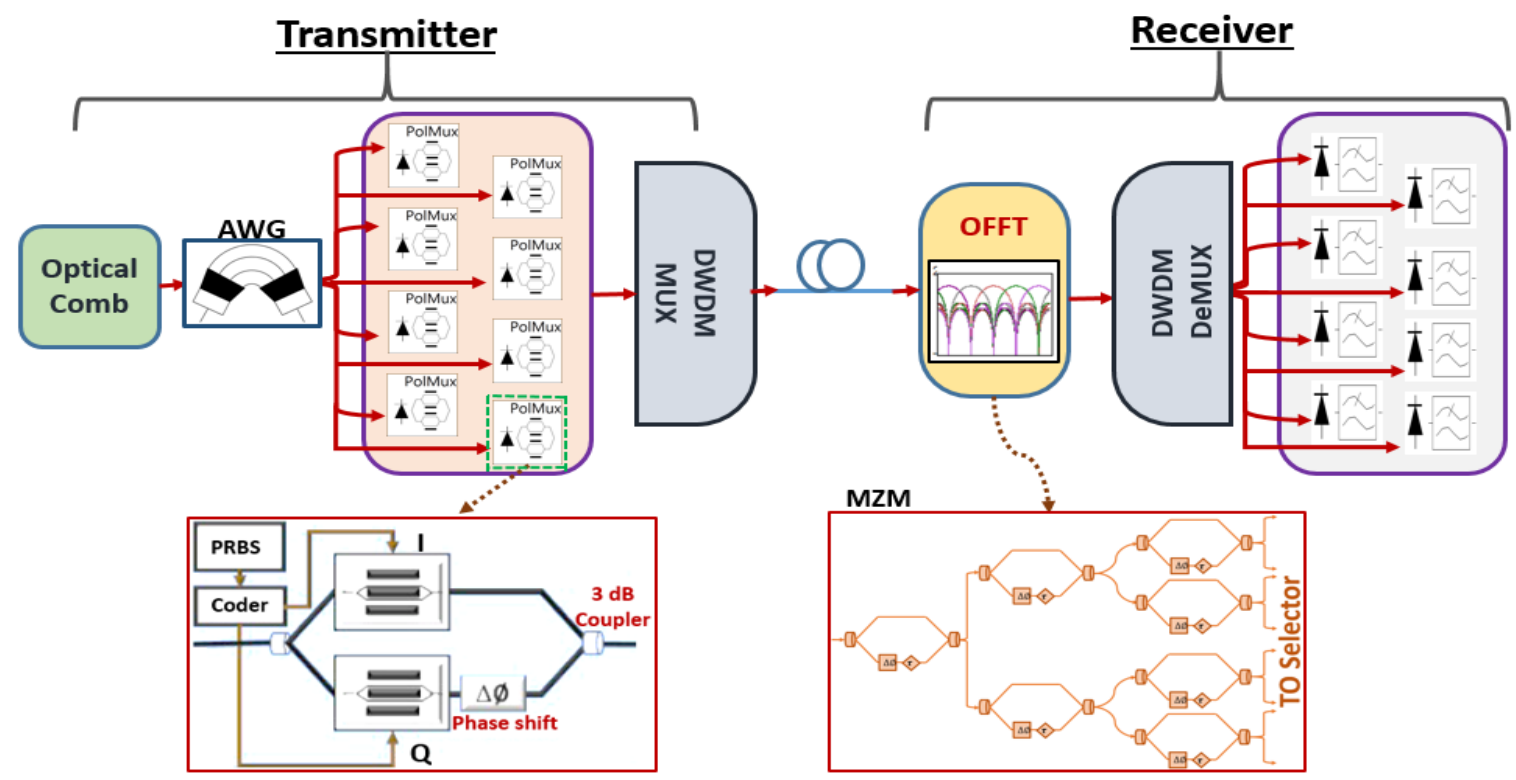

Figure. 2 Schematic of all optical OFDM

Table 1. Parameter of the proposed system

\begin{tabular}{|l|l|}
\hline Parameter & Values \\
\hline Data rate & $240 \mathrm{~Gb} / \mathrm{s}$ \\
\hline $\begin{array}{l}\text { The operating system } \\
\text { for centre frequency }\end{array}$ & $193.1 \mathrm{THz}$ \\
\hline Link range & $(100-1300) \mathrm{km}$ \\
\hline Channel spacing & $20 \mathrm{GHz}$ \\
\hline Modulation technique & QAM, QPSK \\
\hline Laser type & $\mathrm{CW}$ laser \\
\hline Bits per symbol & $\begin{array}{l}3 \text { for QAM } \\
2 \text { for QPSK }\end{array}$ \\
\hline AWG channels & 8 \\
\hline
\end{tabular}

the transmitted operation should be reversed in the receiver side to recover the data from the source. The OFFT circuit is composed of a cascaded MachZehnder interferometer (MZI). Each MZI's lower arm includes a phase shifter and optical time delayer. The first time delay for the MZI is changed to $T_{S} / 2$, while the time delay for two more parallel MZI is set to $T_{s} / 4$, later the parallel four is set to $T_{2} / 8$. The phase shift is set to $\pi / 2 \mathrm{rad}$. The output of $8 \mathrm{MZM}$ is selected by a selector collected all IQ signals. DWDM demultiplexer is used directly to spill the subcarriers. The output from each De DWDM is subsequently filtered by the optical band-pass filter and then detected using an optical demodulator (QAM or QPSK).

\section{Simulation results and discussion}

The results and discussion of high bit rate AWGAO-OFDM will be discussed here, the system investigated to test its performance using QAM and QPSK modulation formats under different input power and transmission lengths. A simulation program called VPI Transmission maker software package was adopted to achieve this. This program offers a precise physical approach to the simulated network and speeds up the design for a high bit rate, long-haul transmission links using various simulation techniques, having large components on the transmitter and receiver side. To explore the scenario for the optical OFDM signal created and detected in the AO-OFDM system, the transmitterside signal generation and the receiver-side signal detection are plotted in Figs. 3 and 4, respectively.

The optical frequency comb source on the transmitter side optically provided 8 comb lines with a frequency spacing of $20 \mathrm{GHz}$, as shown in Fig. 3 (a). The use an optical demultiplexer after splitting the comb spectrum into single comb lines called subcarriers, as shown in Fig. 3 (b). These subcarriers were carry over to AWG that feeds a single wavelength into each modulator to modulated independently using optical QAM or QPSK modulator, as illustrated in Fig. 3 (c). Each separated comb line was modulated at a symbol rate equal to $240 \mathrm{~Gb} / \mathrm{s}$. At this point, the total data rate is equal to $2 \times 8 \times 240=3.8 \mathrm{~Tb} / \mathrm{s}$. Two independent pseudo-random binary sequence (PRBS) signals were produced for the modulation symbols for each separate comb line, each having a length of $\left(2^{11}-1\right)$ bits using a QAM and QPSK encoder, that both generates the components in-phase and quadrature. Every one of the separate comb lines is divided by a $3 \mathrm{~dB}$ coupler and inserted into two parallel MZM. On the receiver side, the optical OFDM signal 


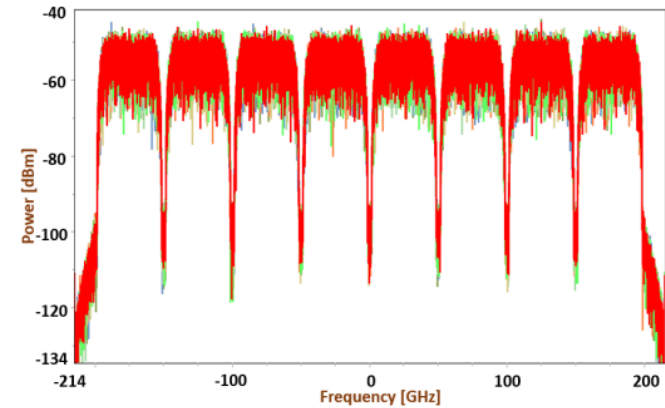

(a)

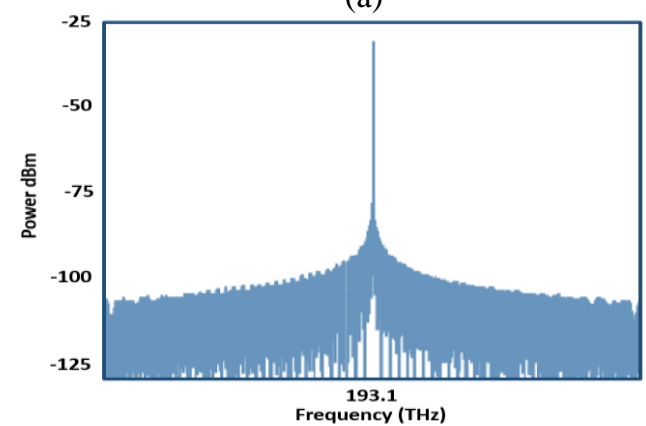

(b)

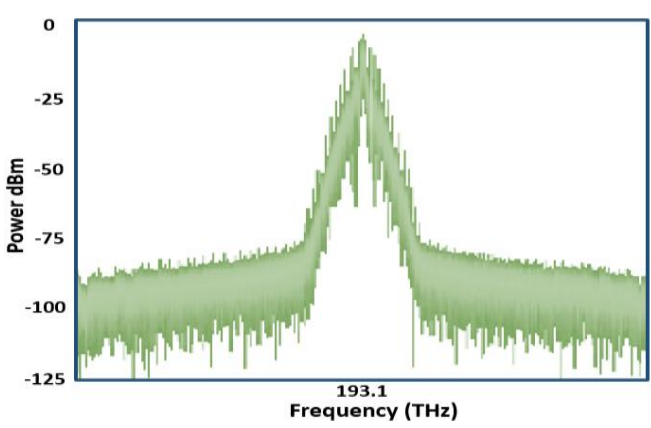

(c)

Figure. 3 Optical scope spectrum for the received signals at the transmitter: (a) 8 comb line, (b) after separate comb line, and (c) signal of QAM and QPSK

received is processed using an optical FFT circuit as shown in Fig. 4.

When the orthogonally, transmitting and received signal has been achieved, studying the effect of both input power and transmitting distance into the performance of this system and demonstrating the difference between the two advance modulations used.

The constellation diagram defines the digitally modulated signal, which is described as a dispersion diagram in two dimensions. Fig. 5 depicts the signal constellation diagram of the 8 QAM system after using the different input power. Fig. 5 (a) shown amplitude of the constellation OFDM signal using $2 \mathrm{dBm}$ input power. The maximum and minimum amplitude value is 4.34 (a.u) and -4.18 (a.u). While when using $0 \mathrm{dBm}$ input power, the amplitude value is 4.43 (a.u) and -4.08 (a.u) in Fig. 5 (b). That's means, when increases the input power, the

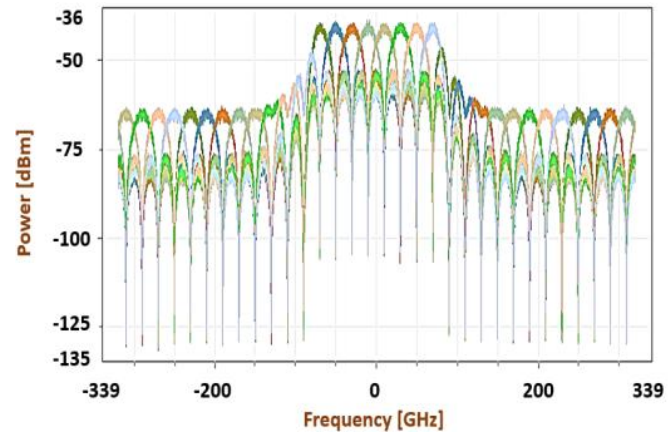

Figure. 4 Optical spectrum analyzer after using an optical FFT

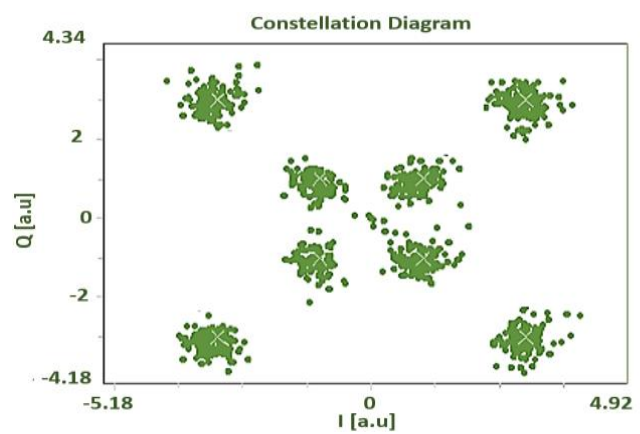

(a)

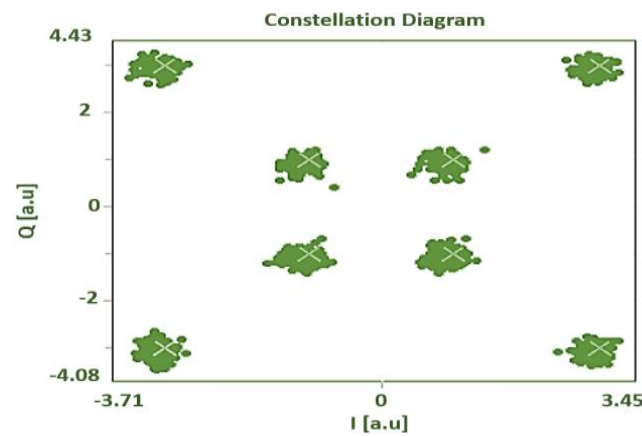

(b)

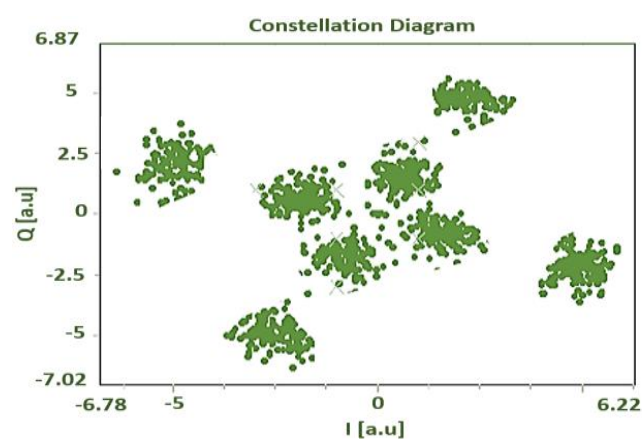

(c)

Figure. 5 Received constellation diagrams for 8 QAM for channel 4 with: (a) $-2 \mathrm{dBm}$, (b) $0 \mathrm{dBm}$, and (c) $2 \mathrm{dBm}$

amplitude value of the OFDM signal is increased and reached the best value in $2 \mathrm{dBm}$ input power in Fig. 5 (c) with 6.87 (a.u) and -7.02 (a.u) maximum and minimum amplitude value. Based on the measurement of the constellation OFDM signal, if low input power values are set, signal distortion is 
high. Furthermore, according to the nonlinear effect of self-phase modulation (SPM), setting a higher input power would result in a greater distortion of nonlinearity. Transmission tests were obtained for a central channel as this has the highest rate of nonlinearity.

Fig. 6 illustrated the signal constellation of 8 QAM after different transmission distance with optical fiber length $100 \mathrm{~km}, 700 \mathrm{~km}$, and $1300 \mathrm{~km}$. The transmission checks for a central DWDM channel were achieved. Fig. 6 (a) shown the maximum and minimum amplitude of the constellation OFDM signal is 3.61 (a.u) and -3.81 (a.u), while is 3.88 (a.u) and -4.13 (a.u) in Fig. 6 (b). That means the maximum amplitude increases after increased transmission distance and this amplitude is reached the maximum value in Fig. 6 (c) which is 4.83 (a.u) and -4.91 (a.u) at $(1300 \mathrm{~km})$ for $0 \mathrm{dBm}$ input power.
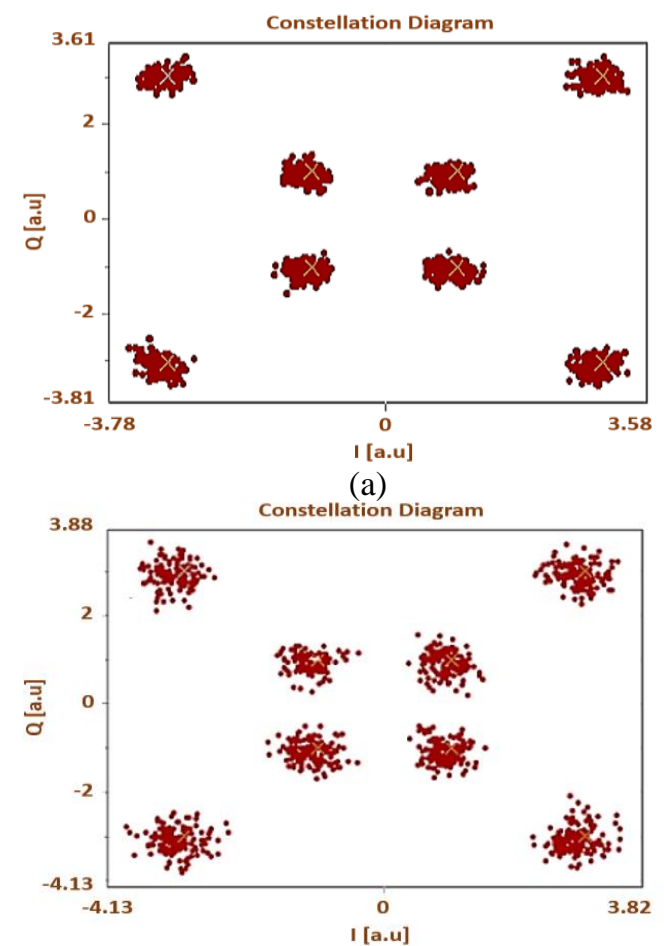

(b)

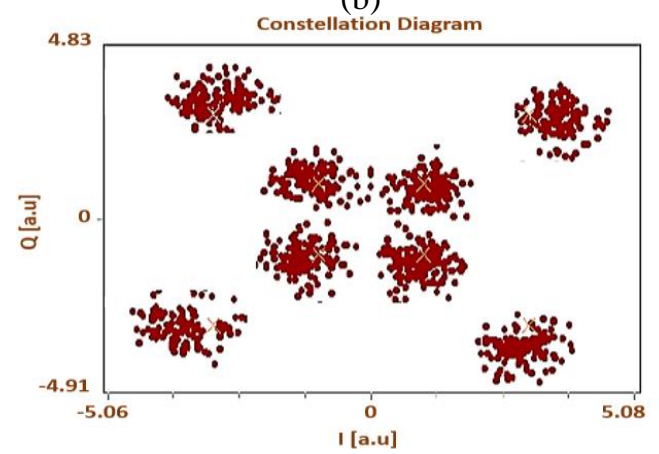

(c)

Figure. 6 Received constellation diagrams for 8 QAM for channel 4 at: (a) $100 \mathrm{~km}$, (b) $700 \mathrm{~km}$, and (c) $1300 \mathrm{~km}$

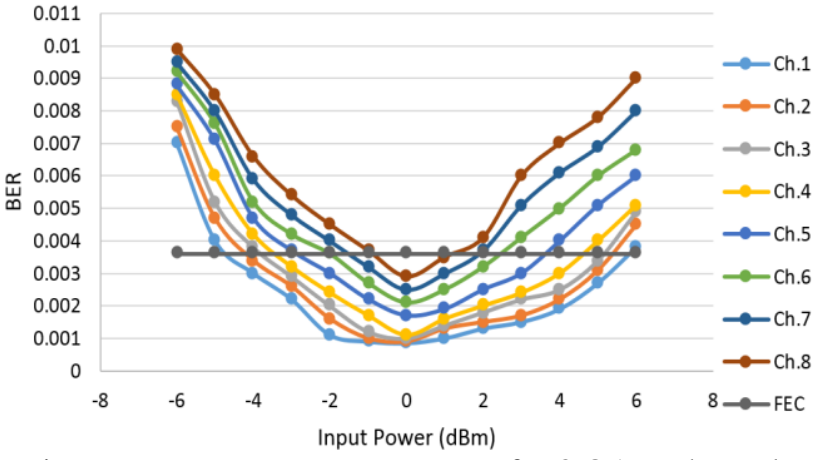

Figure. 7 Input power versus BER for 8 QAM channels

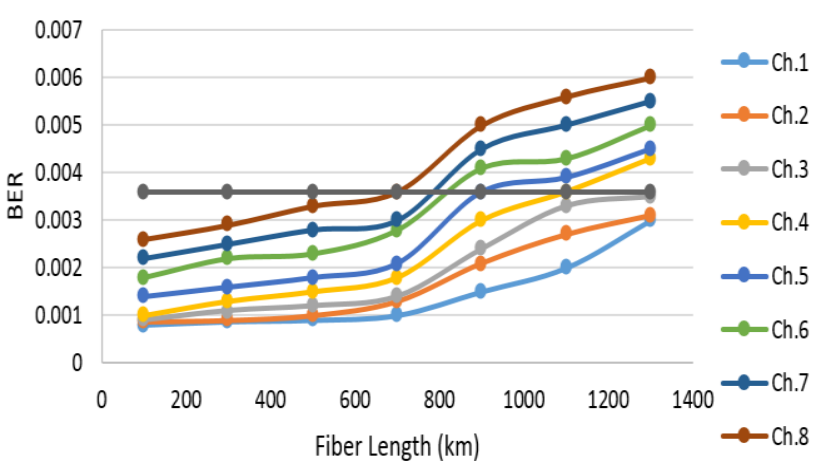

Figure. 8 Length of fiber versus BER for 8 QAM channels

The performance of AO-OFDM based AWG is achieved by measuring BER as a function of the input power and the transmission length for two modulation formats.

Fig. 7 demonstrated the relationship between the input power versus BER for 8 QAM modulation format where the input power ranges from $-6 \mathrm{dBm}$ to $6 \mathrm{dBm}$. As can be seen, the BER decreases as the input power increase. However, as the input power decreases beyond $1 \mathrm{dBm}$ to $-1 \mathrm{dBm}$, the BER increase dramatically achieved at the threshold BER $=10^{-3}$.

Fig. 8 depicts the BER as a function of fiber length. Similarly, if the fiber length increases from $100 \mathrm{~km}$ to $1300 \mathrm{~km}$, the BER decrease, until the fiber length reached $750 \mathrm{~km}$, the minimum BER is obtained to $10^{-3}$ at an input power of $-1 \mathrm{dBm}$ to 1 $\mathrm{dBm}$.

In the QPSK system, Fig. 9 displays the constellation diagrams as indicated in the QAM system at different input power at $(-4,0,4) \mathrm{dBm}$ for a specific DWDM channel (channel four). Fig. 9 (a) shown the maximum and minimum amplitude of output constellation diagram of the OFDM signal that is 1.46 (a.u) and -1.58 (a.u) at $-4 \mathrm{dBm}$ input power. This amplitude value is increased to 1.49 (a.u) maximum value and -1.46 (a.u) minimum value in $0 \mathrm{dBm}$ input power in Fig. 9 (b), while it 
becomes the largest value in $4 \mathrm{dBm}$ input value as shown in Fig. 9 (c).

The QPSK designed method supported for long haul transmission. The QPSK system obtained constellation diagrams with different length as shown in Fig. 10. Fig. 10 (a) illustrated of constellation OFDM signal at $100 \mathrm{~km}$ transmission length. The amplitude value at maximum and minimum is 1.35 (a.u) and -1.48 (a.u), while at 700 $\mathrm{km}$ is 1.61 (a.u) and -1.64 (a.u). That is meaning the transmission length can be increased without effect on the output signal of OFDM at $0 \mathrm{dBm}$ input power. For this, we can increase the transmission length to $1300 \mathrm{~km}$ to get maximum and minimum amplitude value 1.99 (a.u) and -2.13 (a.u) as shown in Fig. 10 (c).

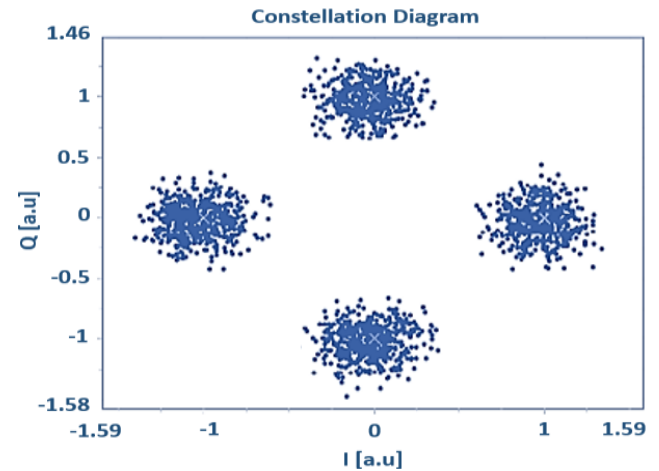

(a)

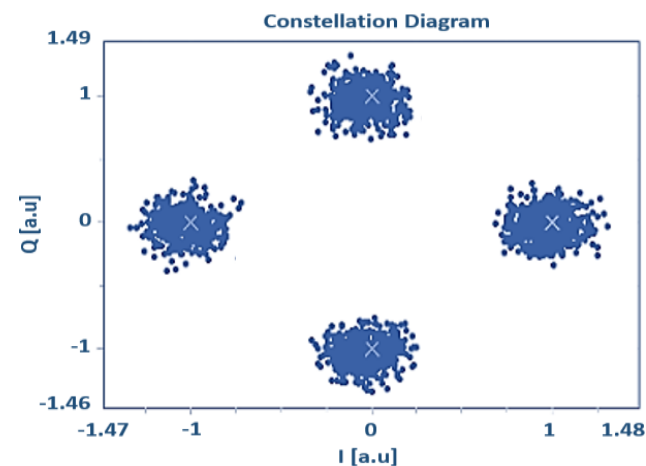

(b)

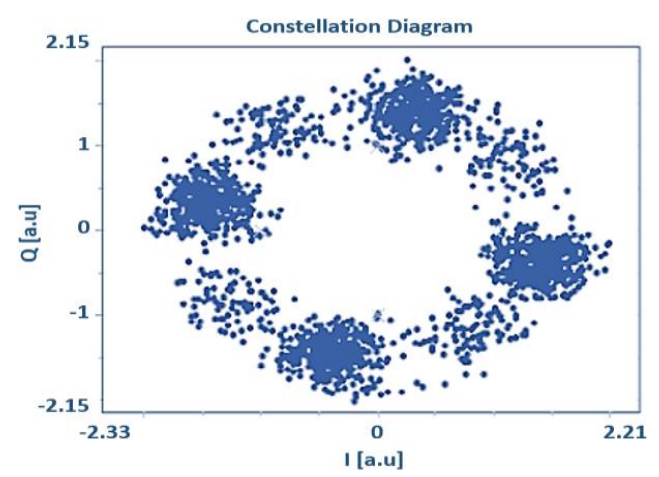

(c)

Figure. 9 Received constellation diagrams for QPSK of channel 4 with: (a) $-4 \mathrm{dBm}$, (b) $0 \mathrm{dBm}$, and (c) $4 \mathrm{dBm}$

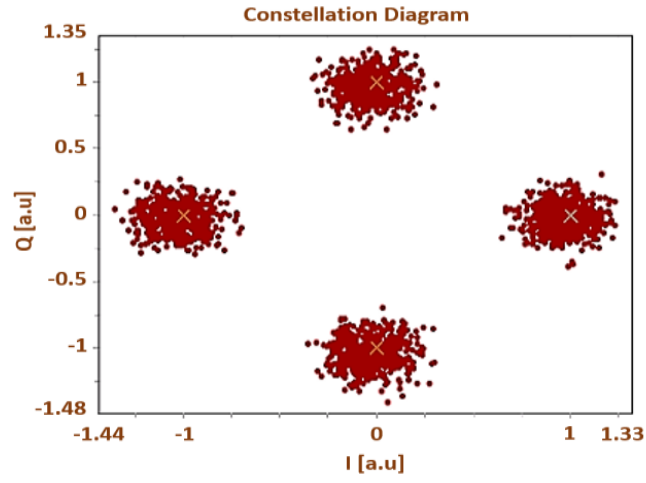

(a)

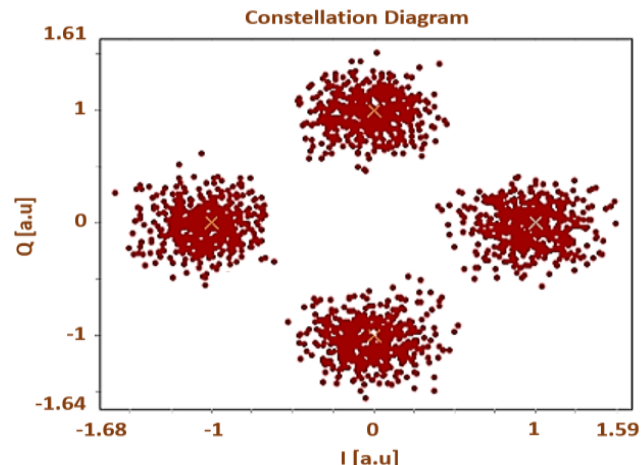

(b)

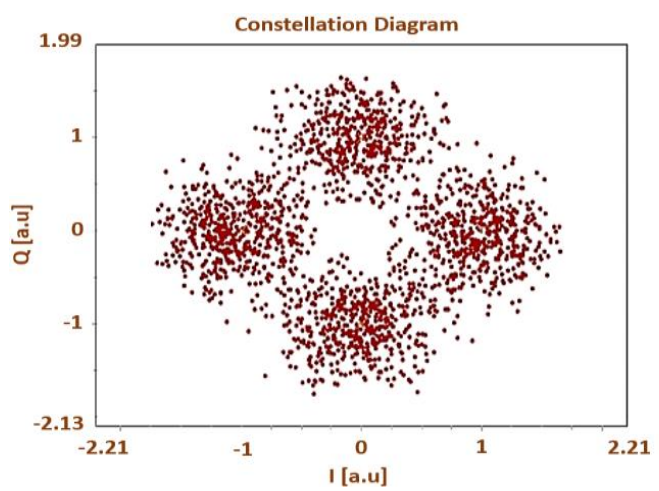

(c)

Figure. 10 Received constellation diagrams for QPSK for channel 4 at: (a) $100 \mathrm{~km}$, (b) $700 \mathrm{~km}$, and (c) $1300 \mathrm{~km}$

Regarding the QPSK system, the performance is also evaluated by calculating BER as a function of input power and length of the fiber. Fig. 11 illustrated the BER as a function of input power. The input power is varied from $6 \mathrm{dBm}$ to $-6 \mathrm{dBm}$. As is obvious, as the input power increases, the BER decreases until the input power reaches $3 \mathrm{dBm}$ and $3 \mathrm{dBm}$ to achieved $10^{-3}$.

Fig. 12 shows the relation between fiber length versus BER where the length of the transmission ranges from $100 \mathrm{~km}$ to $1300 \mathrm{~km}$. The BER, as demonstrated, gets threshold value $10^{-3}$ when reaching to $1100 \mathrm{~km}$ at an input power of $-3 \mathrm{dBm}$ to $3 \mathrm{dBm}$. 
Received: March 13, 2020. Revised: April 15, 2020.

Table 2. Comparison of proposed work with previously published work

\begin{tabular}{|c|c|c|c|c|c|c|c|c|}
\hline Parameters & In [7] & In [8] & In [9] & In [10] & In [11] & In [13] & In [15] & $\begin{array}{c}\text { Proposed } \\
\text { work }\end{array}$ \\
\hline Data rate (Gb/s) & 25 & 120 & 400 & 160 & 100 & 100 & $\begin{array}{c}458 \text { per } \\
\text { single } \\
\text { polarize }\end{array}$ & 240 \\
\hline $\begin{array}{c}\text { Transmission } \\
\text { distance (km) }\end{array}$ & 40 & 800 & ------ & ------ & 25 & 900 & ------ & 1100 \\
\hline $\begin{array}{c}\text { Modulation format } \\
\text { QPSK }\end{array}$ & QPSK & $\begin{array}{l}\text { QAM, } \\
\text { QPSK }\end{array}$ & QPSK & $\begin{array}{c}\text { DPSK, } \\
\text { PAM, } \\
\text { QAM }\end{array}$ & QAM & QPSK & $\begin{array}{c}\text { QAM, } \\
\text { QPSK }\end{array}$ \\
\hline $\begin{array}{c}\text { Channel spacing } \\
\text { (GHz) }\end{array}$ & ------- & 40 & 50 & 50 & 50 & ------- & 200 & 20 \\
\hline $\begin{array}{c}\text { Multiplexing type } \\
\text { Channel input } \\
\text { power (dBm) }\end{array}$ & AWG & DWDM & WDM & DWDM & AWG & ------- & AWG & $\begin{array}{c}\text { AWG + } \\
\text { DWDM }\end{array}$ \\
\hline No. of channels & 64 & 15 & 4 & 16 & 8 & -------- & 16 & 8 \\
\hline BER & $10^{-3}$ & -------- & -------- & $3.8 \times 10^{-3}$ & $10^{-3}$ & $7.3 \times 10^{-3}$ & ------- & $3.8 \times 10^{-3}$ \\
\hline Total bit rate (Tb/s) & 1.6 & 1.8 & 1.6 & 1.5 & 0.8 & 1 & 1.4 & 3.8 \\
\hline
\end{tabular}

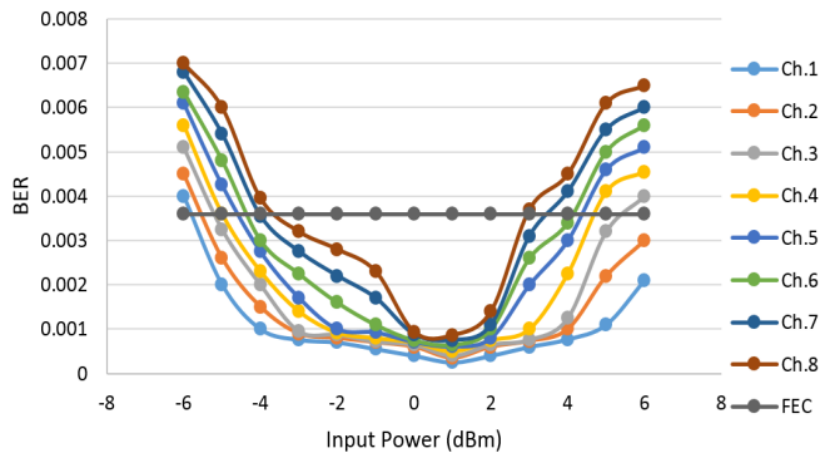

Figure. 11 Input power versus BER for 8 QPSK channels

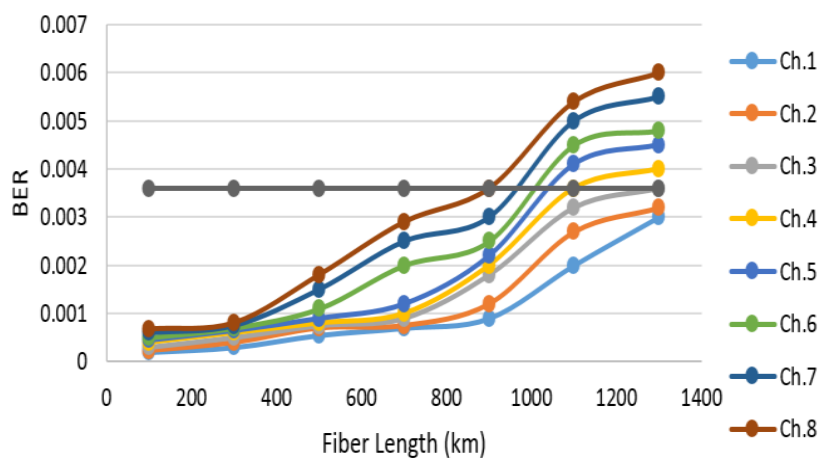

Figure. 12 Length of fiber versus BER for 8 QPSK channels

Generally, the AO-OFDM system with QPSK has a better BER and transmission length than the QAM modulation format with a total data rate equal to $3.8 \mathrm{~Tb} / \mathrm{s}$.

Table 2 provides a comparison of the current study with previously published work of all optical OFDM systems. After the evaluation and validation of the proposed system, it is compared with related works presented by $[7-11,13,15]$ as shown in Table 2. The FFT and IFFT function achieved using the AWG device. The AWG has less systemic complexity compared to other methods, in particular for a large $\mathrm{N}$ subcarrier. Furthermore, the combined AWG device with the DWDM technique could be covered to solve the problem of nonlinearity distortion using all optical transmission. Reference [7] designed an optical circuit based on multiplexing and coherent detection, for passive optical networks multi terabit per second achieving higher-order fast fourier transform functions by arranging multiple fast fourier transform circuits in a smaller order. Yet the complexity of the circuit is increasing rapidly, as $\mathrm{N}$ increases. By comparison, the AWG-based IFFT / FFT circuit in the current study needs only the arrayed waveguide and the two slab regions to be built for any size of $\mathrm{N}$. We observed that the best transmission distance is $900 \mathrm{~km}$ in [13], while in the current study reached to $1100 \mathrm{~km}$. The best capacity rate is $1.8 \mathrm{~Tb} / \mathrm{s}$ in [8] using 15 channels, while in this study reached $3.8 \mathrm{~Tb} / \mathrm{s}$ using 8 channels only. Any previous study could not overcome the channel spacing and reduce it by more than $40 \mathrm{GHz}$ due to nonlinearity effects, whereas in this study is reduced to $20 \mathrm{GHz}$ without affecting the quality of the received signal.

\section{Conclusion}

In this study, we successfully designed a new method to generate all optical OFDM simulation systems using arrayed waveguide gratings. This 
method comprises new technology intended to break the barrier of data limits. The system was analyzed under various input powers, lengths and modulation formats. The use of the AWG and DWDM technique with $20 \mathrm{GHz}$ channel spacing, has resulted in a total bit rate of up to $3.8 \mathrm{~Tb} / \mathrm{s}$ with two modulation formats. The constellation diagrams and BER performance of the received signals are measured at the receiver for the central channel, as the norm of the highest nonlinearity quality. The maximum distance in QAM format is $750 \mathrm{~km}$ with an input power range from $-1 \mathrm{dBm}$ to $1 \mathrm{dBm}$, while in QPSK format, it is $1100 \mathrm{~km}$ with an appropriate input range of $-3 \mathrm{dBm}$ to $3 \mathrm{dBm}$. The results show that QPSK-AO-OFDM gave the best performance with a high data rate and long transmission distance.

It is suggested that future work looks to develop ultra-high capacity transmission systems using DWDM-SDM techniques with different modulation formats. Another line of investigation would be to increase the number of DWDM channels to study the effect of nonlinearity.

\section{Conflicts of Interest}

The author declares no conflict of interest.

\section{Author Contributions}

Ali Hayder Abdul Kareem: conceptualization, methodology, software, validation, formal analysis, investigation, resources, data curation, writingoriginal draft preparation, writing-review and editing, visualization, supervision, project administration, and funding acquisition.

\section{References}

[1] D. Richardson, "New optical fibres for highcapacity optical communications", Philosophical Transactions of the Royal Society A: Mathematical, Physical and Engineering Sciences, Vol. 374, No. 2062, p. 20140441, 2016.

[2] M. Jinno, H. Takara, B. Kozicki, Y. Tsukishima, Y. Sone, and S. Matsuoka, "Spectrum-efficient and scalable elastic optical path network: architecture, benefits, and enabling technologies", IEEE Communications Magazine, Vol. 47, No. 11, pp. 66-73, 2009.

[3] J. He, F. Long, R. Deng, J. Shi, M. Dai, and L. Chen, "Flexible multiband OFDM ultrawideband services based on optical frequency combs", IEEE/OSA Journal of Optical Communications and Networking, Vol. 9, No. 5, pp. 393-400, 2017.
[4] L. Nadal Reixats, "Performance analysis of optical OFDM transmission systems using PAPR mitigation techniques and alternative transforms", Msc Thesis, Universitat Politècnica de Catalunya, 2012.

[5] M. Imran, P. M. Anandarajah, A. Kaszubowska-Anandarajah, N. Sambo, and L. Potí, "A survey of optical carrier generation techniques for terabit capacity elastic optical networks", IEEE Communications Surveys \& Tutorials, Vol. 20, No. 1, pp. 211-263, 2017.

[6] K. Alatawi, F. Almasoudi, and M. A. Matin, "Integration of coherent optical OFDM with WDM", in Optics and Photonics for Information Processing VII, Vol. 8855, p. 88550P, 2013.

[7] J. Morosi, J. Hoxha, P. Martelli, P. Parolari, G. Cincotti, S. Shimizu, N. Wada, and P. Boffi, "25 Gbit/s per user coherent all-optical OFDM for Tbit/s-capable PONs", Journal of Optical Communications and Networking, Vol. 8, No. 4, pp. 190-195, 2016.

[8] B. Song, C. Zhu, B. Corcoran, L. Zhuang, and A. J. Lowery, "Banded all-optical OFDM super-channels with low-bandwidth receivers," Optics express, Vol. 24, No. 16, pp. 1796817979, 2016.

[9] D. Hillerkuss, M. Winter, M. Teschke, A. Marculescu, J. Li, G. Sigurdsson, K. Worms, S. Ben Ezra, N. Narkiss, W. Freude, and J. Leuthold, "Simple all-optical FFT scheme enabling Tbit/s real-time signal processing," Optics express, Vol. 18, No. 9, pp. 9324-9340, 2010.

[10] Z. Geng, D. Kong, B. Corcoran, P. Guan, F. Da Ros, E. P. Da Silva, L. K. Oxenlowe, and A. J. Lowery, "All-optical OFDM demultiplexing with optical partial Fourier transform and coherent sampling", Optics letters, Vol. 44, No. 2, pp. 443-446, 2019.

[11] G. S. Srinivasan and S. Prince, "All optical OFDM transmission system based future optical broadband networks", In: Proc. of 2016 International Conference on Wireless Communications, Signal Processing and Networking (WiSPNET), pp. 1154-1158, 2016.

[12] A. H. A. Kareem, "Performance Evaluation of PSK All optical OFDM System Based on Arrayed Waveguide Grating under Different Weather Conditions", International Journal of Microwave and Optical Technology, Vol. 15, No. 1, pp. 85-94, 2020.

[13] V. N. Sreedevi Prasanna, "1Tbps All-Optical OFDM Transmission Based on Wavelet Transform", International Journal of 
Microwave and Optical Technology, Vol. 12, No. 4, p. 9, 2017.

[14] S. Shimizu, G. Cincotti, and N. Wada, "Demonstration and performance investigation of all-optical OFDM systems based on arrayed waveguide gratings", Optics express, Vol. 20, No. 26, pp. B525-B534, 2012.

[15] A. Abrardo and G. Cincotti, "Design of AWG devices for all-optical time-frequency packing", Journal of lightwave technology, Vol. 32, No. 10, pp. 1951-1959, 2014.

[16] D. Meena, O. S. Sunishkumar, D. C. Pande, T. Srinivas, V. K. Jayasree, F. Francis, K. T. Sarath, and E. Dipin, "A Geometrical Model for Arrayed Waveguide Grating based Optical Multiplexer/Demultiplexer", Progress in Electromagnetics Research, Vol. 35, pp. 87-96, 2014.

[17] V. Shukla, A. Jain, and R. Srivastava, "Performance evaluation of an AWG based optical router", Optical and Quantum Electronics, Vol. 48, No. 1, p. 69, 2016.

[18] Z. Wang, K. S. Kravtsov, Y.-K. Huang, and P. R. Prucnal, "Optical FFT/IFFT circuit realization using arrayed waveguide gratings and the applications in all-optical OFDM system", Optics Express, Vol. 19, No. 5, pp. 4501-4512, 2011. 